\title{
800nm fiber Bragg grating sensing interrogation system using TFBG and CCD array
}

Bo Liu, Rui Suo, Kaiming Zhou, Long Jin, Jian Zhang, et al.

Bo Liu, Rui Suo, Kaiming Zhou, Long Jin, Jian Zhang, Yange Liu, Guiyun Kai, Xiaoyi Dong, "800nm fiber Bragg grating sensing interrogation system using TFBG and CCD array," Proc. SPIE 6595, Fundamental Problems of Optoelectronics and Microelectronics III, 659545 (5 March 2007); doi: 10.1117/12.726504

SPIE Event: Fundamental Problems of Optoelectronics and Microelectronics III, 2006, Harbin, China 


\title{
800nm Fiber Bragg Grating Sensing Interrogation System Using TFBG and CCD array
}

\author{
Bo Liu(1), Rui Suo(2), Kaiming Zhou(2), Long Jin(1), Jian Zhang(1), \\ Yange Liu(1), Guiyun Kai(1), Xiaoyi Dong(1) \\ 1 : Institute of Modern Optics, Nankai Univ. Tianjin, P.R.China, 300071, \\ liubo@mail.nankai.edu.cn \\ 2 : Photonics Research Group, ASTON Univ. Birmingham, UK, B4 7ET
}

\begin{abstract}
:
An $800 \mathrm{~nm}$ band fiber Bragg grating sensing interrogation system using TFBG as the core wavelength division component is presented. A charge coupled device (CCD) linear array is put on the focal plane of the lens to detect the light. TFBG is used to tap light out of the fiber core to fiber cladding. The sensing wavelength is 795 to $830 \mathrm{~nm}$, with accuracy of $20 \mathrm{pm}$ and scan speed $100 \mathrm{~Hz}$. Using FBG sensor, we achieve the temperature sensitivity as $1.8^{\circ} \mathrm{C}$ and strain sensitivity as $18 \mu \varepsilon$.
\end{abstract}

Keywords : TFBG, BFBG, interrogation system, FBG sensing

\section{INTRODUCTION}

In recent years, fiber grating sensor techniques are attracting enormous interest because of their super advantages such as inherent wavelength-encoded operation, low cost, high accuracy and small size. Thus, it is important to develop a simple system to measure the spectrum. ${ }^{[1-4]}$

Tilted fiber Bragg grating (TFBG) is a special kind of fiber grating that there is a certain angle between the grating plane and fiber axis, which is first reported in 1990 by Meltz et al., and has been demonstrated for applications. TFBG converts forward propagating modes to a combination of forward, backward and radiation modes with the ratio determined by several fabrication parameters but most importantly by tilted angle.

In this paper, we present an $800 \mathrm{~nm}$ band fiber Bragg grating sensing interrogation system using TFBG as the core wavelength division component. A charge coupled device (CCD) linear array is put on the focal plane to detect the light. Different sensing pixels on CCD array will sense different wavelength of light. The sensing wavelength is 795 to $830 \mathrm{~nm}$, with accuracy of $20 \mathrm{pm}$ and scan speed $100 \mathrm{~Hz}$. Using FBG sensor, we achieve the temperature sensitivity as $1.8^{\circ} \mathrm{C}$ and strain sensitivity as $18 \mu \varepsilon$.

Fundamental Problems of Optoelectronics and Microelectronics III edited by Yuri N. Kulchin, Jinping Ou, Oleg B. Vitrik, Zhi Zhou

Proc. of SPIE Vol. 6595, 659545, (2007) · 0277-786X/07/\$18 - doi: 10.1117/12.726504

Proc. of SPIE Vol. 6595 659545-1 


\section{BASIC PRINCIPLE}

The TFBG used in this system is written in holographic method, with 488nm laser, as shown in Fig.1.

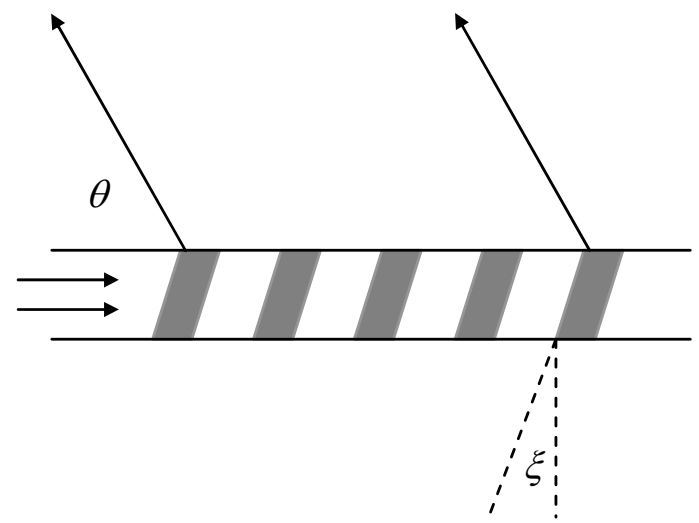

Fig. 1 Structure of TFBG

$\theta$ is the angle between coupling out light and the fiber axis. $\xi$ is the angle between the grating plane and fiber axis. Using Coupling Method, we can get the propagation equation of TFBG as follows ${ }^{[5-6]}$.

$$
\cos (\theta)=\frac{\frac{\lambda}{\Lambda} \cos (\xi)-n_{\text {eff }}}{n_{\text {clad }}}
$$

Where $\lambda$ is the light wavelength, $\Lambda$ is the period of TFBG, $n_{\text {eff }}$ is the efficiency index of fiber core, and $n_{\text {clad }}$ is the index of fiber clad. ${ }^{[7-9]}$

\section{EXPERIMENTAL SYSTEM}

The scheme of TFBG interrogation system is shown in Fig.2. Light from broad band source (BBS) irradiates into the sensing fiber Bragg grating (FBG) via a $3 \mathrm{~dB}$ coupler. Then the reflect light from FBG irradiates into TFBG. TFBG is put into index match gel (IMG) in order to couple the light which propagates in fiber clad by TFBG out of the fiber. 


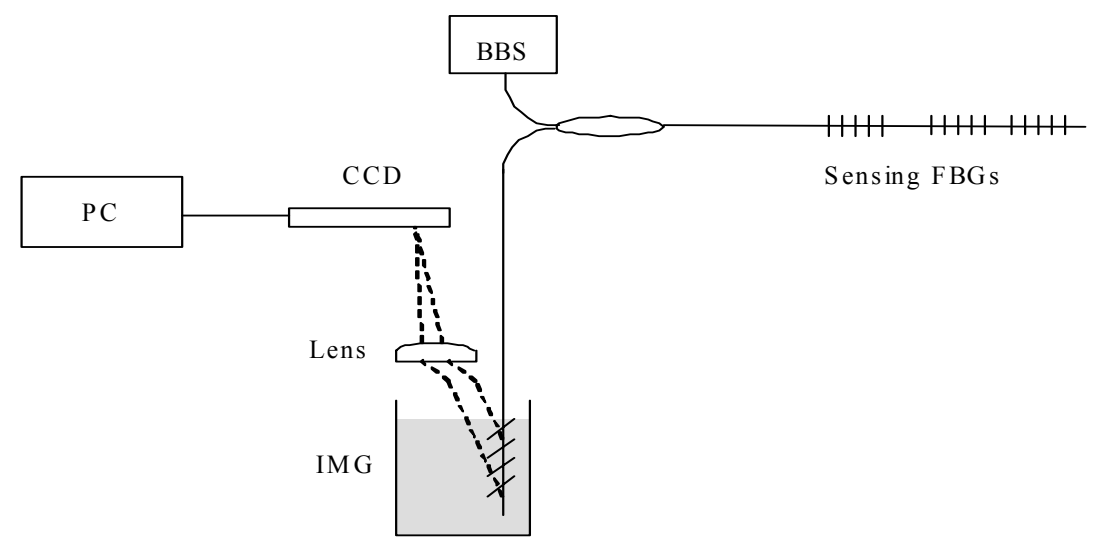

Fig.2 Scheme of TFBG interrogation system

From equation (1), we know that lights with the same wavelength will go out of fiber parallelly. So a lens is used to focus the parallel lights to one point. Also from equation (1), we know that lights with different wavelength have different $\theta$. And they will focus to different points which are on focal plane of the lens. Thus, a CCD linear array is put there to detect the light. Different sensing pixels on CCD array will present different wavelength of light. Fig.3 shows the practical system we build.

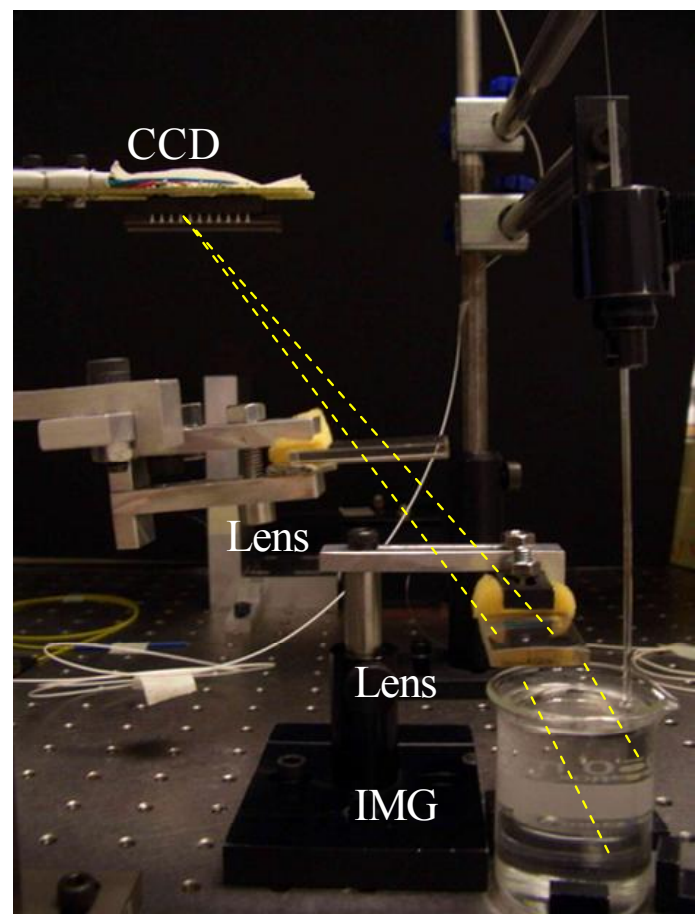

Fig.3 Practical system of TFBG 
The transmissive spectrum of the TFBG includes $795 \mathrm{~nm}$ to $830 \mathrm{~nm}$, as shown in Fig.4. We use SONYILX511 CCD linear array as light detecting element, which is 2048 pixels and $14 \mu \mathrm{m}$ length per pixel. The response wavelength range of the CCD array is $400 \mathrm{~nm}$ to $1000 \mathrm{~nm}$, so it needs not additional coat which cost a lot. An $800 \mathrm{~nm}$ SLD is used as broad band source to supply light.

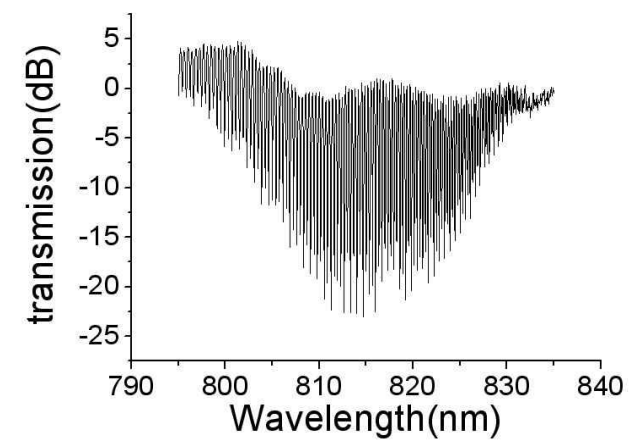

Fig.4 Transmissive spectrum of TFBG

\section{EXPERIMENTAL RESULT}

In the experiment, an $819 \mathrm{~nm}$ FBG as sensing component is connected to the output of SLD. The transmissive port the sensing FBG is connected to TFBG. The light is radiated onto the CCD array and the waveform is captured by computer. In this way, strain and temperature sensing is proceeded. The experimental results show that the accuracy of this interrogation system is about $25 \mathrm{pm}$, and the sensing range is about $40 \mathrm{~nm}$. We set the integral time of CCD as $10 \mathrm{~ms}$.

First we change the temperature of sensing FBG from $-10^{\circ} \mathrm{C}$ to $60^{\circ} \mathrm{C}$. Fig. 5 shows the relationship between temperature and peak position of CCD data. The accuracy of temperature of this system is about $1.8^{\circ} \mathrm{C}$.

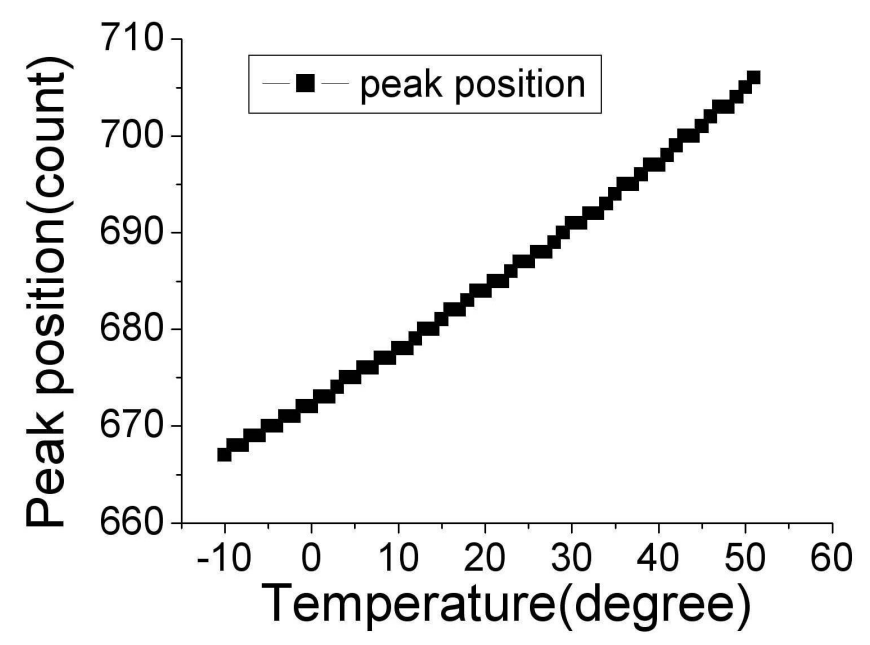

Fig.5 Temperature vs peak position 
Then we change the strain of sensing FBG from 0 to $1500 \mu \varepsilon$. Fig. 6 shows the relationship between strain peak position of CCD data.

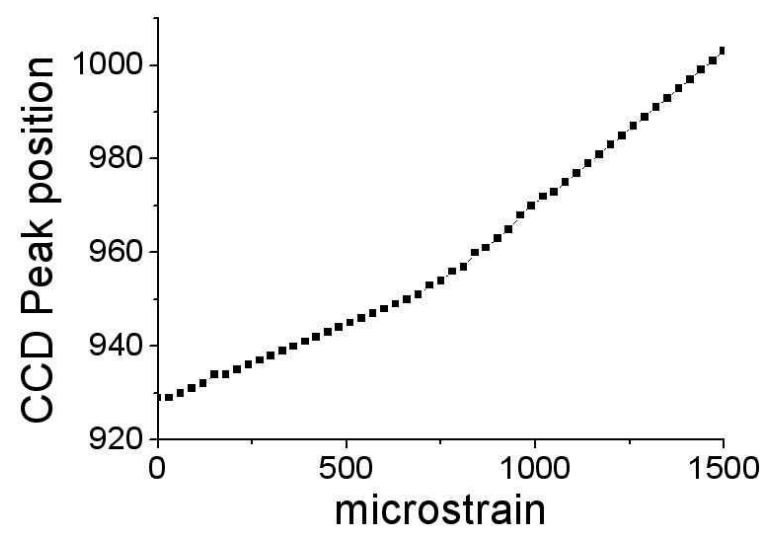

Fig.6 Strain vs peak position

The accuracy of strain of this system is about $18 \mu \varepsilon$.

\section{CONCLUSION}

This interrogation system is based on TFBG and CCD linear array. Because there is none mechanical part, it has high stability and high response frequency which only depend on CCD integrate time (ms level). It will be widely used in real-time wavelength monitor.

\section{Acknowledgments}

Support by the Chinese National Science Fund: 60577018

\section{REFERENCES}

1. Bai-Ou Guan, et al., IEEE Photon. Technol. Lett., 2000, 12(6):675-677

2. Dejun Feng, et al., Proceedings of SPIE, Vladivostok, Russia, Sept, 11-15,2000, Vol.4513:7-11

3. T. Coroy, et al., Electron Lett., 1996, 32(19): 1811-1812

4. R.P. Moeller, et al., Electron. Lett., 2001,37(1):14-15

5. K.Zhou. Aston University, UK. Unpublished work

6. Yufeng Li, et al., JOURNAL OF LIGHTWAVE TECHNOLOGY, OCTOBER 2001, 19(10): 1580-1591

7. AG. Simpson, et al., Applied Optics, 2004, 43(1): 33-40

8. K. Zhou, et al., CLEO, 2003, Baltimore, USA

9. K. Zhou, et al., IEEE Photonics Technology Letters, 2003, 15(7): 936-938 